Research Paper

\title{
Gross Total Resection of Glioma with the Intraoperative Fluorescence- guidance of Fluorescein Sodium
}

\section{Bo Chen ${ }^{1 *}$, Haifeng Wang ${ }^{1 *}$, Pengfei Ge1${ }^{1}$, Jingwei Zhao ${ }^{3}$, Wenchen $\mathrm{Li}^{1}$, Huizi Gu², Guangming Wang ${ }^{1}$, Yinan Luo ${ }^{1 凶}$, Dawei Chen ${ }^{1 凶}$}

1. Department of neurosurgery, The First Hospital of Jilin University, Changchun, Jilin, 130021, China

2. Department of neuroscience, The First Hospital of Jilin University, Changchun, Jilin, 130021, China

3. Department of neurosurgery, The China-Japan Union Hospital of Jilin University, Changchun, Jilin, 130033, China

${ }^{*}$ Contributed equally as the co-first author.

Corresponding author: Dr. Dawei Chen and Dr. Yinan Luo, Department of neurosurgery, The First Hospital of Jilin University. \#71 Xinmin Street, Changchun, Jilin, 130021, P.R. China. Tel: +86-13074366977, E-mail: dchenjdyy@gmail.com or yluojdyy@gmail.com

(c) Ivyspring International Publisher. This is an open-access article distributed under the terms of the Creative Commons License (http://creativecommons.org/ licenses/by-nc-nd/3.0/). Reproduction is permitted for personal, noncommercial use, provided that the article is in whole, unmodified, and properly cited.

Received: 2012.07.09; Accepted: 2012.09.17; Published: 2012.10.06

\begin{abstract}
Objective. High dose fluorescein sodium has been utilized for fluorescence-guided tumor resection with conflicting reports on the efficacy of this procedure. The aim of this study was to reevaluate the utility and clinical limitations of using fluorescein sodium for the treatment and resection of glioma brain tumors.

Methods. Patients diagnosed with glioma were divided into two groups with a total of 22 patients enrolled in the study: I) the study group $(n=10)$, patients that received intravenous injection of fluorescein sodium and 2) the control group ( $n=12)$, patients that did not receive injections during surgical resection. Quality of life was evaluated according to Karnofsky Performance Scale (KPS) score and neurological status. Fluorescein sodium was intravenously injected at a dose of $15-20 \mathrm{mg} / \mathrm{kg}$ of body weight. Glioma resection was evaluated preoperative and postoperatively with enhanced Magnetic Resonance Imaging (MRI).

Results. Significant differences in the gross total resection (GTR) rates were observed between the two patient groups (Fisher's Exact Test $p=0.047$ ). Progressive free survival was significantly longer in the study group (Student's T-Test $p=0.033$ ) as well as in the GTR group (Student's T-Test $p=0.000 \mathrm{I}$ ) compared to the control and non-GTR groups, respectively. Three patients in the study group and four patients in the control group had transient neurological deterioration. One patient in the control group had permanent hemiplegia.

Conclusion. The intraoperative utility of using fluorescein sodium can significantly increase the GTR rate without obvious deterioration. In addition, we find that it is better to apply the fluorescein sodium in the cases with BBB (blood-brain barrier) disruption, which had been enhanced in preoperative MRI.
\end{abstract}

Key words: fluorescein sodium, glioma, gross-total resection, neurosurgery

\section{Introduction}

Glioma is the most common primary malignant brain tumor [1], with poor prognosis to the tumor patient when present at high grades. One and two-year survival rates of high grade glioma patients are only $53.7 \%$ and $14.6 \%$ [2], respectively. Neurosurgery still remains the first-line of treatment [1] as gross tumor resection (GTR) has been reported to be one of the most important factors affecting the prog- 
nosis and survival rate of glioma patients [3, 4]. However, increasing GTR remains a serious challenge to neurosurgeons as it is difficult to distinguish the tumor boundary from normal brain parenchyma due to the infiltrating nature of gliomas $[5,6]$. Accumulating data suggest that intraoperative application of high dose fluorescein sodium guided resection could significantly increase the GTR rate $[5,7]$ while other reports questioned the usefulness of this technique [8, 9]. In the present study, we reevaluate the utility of fluorescein sodium-guided surgery in gliomas.

\section{Clinical Materials and Methods}

\section{Patient population}

The study approval was granted by the Ethics Committee of The First Hospital of Jilin University. Every patient was informed about the benefits and risks of the study, all the patients had signed the written informed consent. To generate a homogeneous group, factors with potential influence on the results were all considered; including percentages of high-grade gliomas in each cohort, tumor location, tumor size, KPS score, and blood-brain barrier (BBB) disruption. Although a slight but non-significant trend towards factors leading to distribution bias were apparent in the present study, each cohort, of patients was homogeneous. Exclusion criteria included diabetes, severe heart disease, and special locations such as bilateral location, corpus callosum invasion, midline, basal ganglia, posterior fossa and brain stem, preoperative KPS scores less than 60 , and maximum diameter of tumor $<1 \mathrm{~cm}$ or $>5 \mathrm{~cm}$. A total of 22 patients with gliomas conformed to the conditions of this prospective study from June 2010 to June 2011. Among the twenty-two patients, ten were treated by fluorescence-guided neurosurgical resection with fluorescein sodium (study group) and twelve were treated without the use of fluorescence during neurosurgical resection (control group). In the study group, there were six female patients and four male patients, whose ages ranged from 31 to 67 years. The mean age of patients in the study group was 50.7 \pm 10.4 (median 50 years). Duration of disease ranged between 10 to 180 days (mean 60 days). Preoperative KPS score was $78 \pm 9.2$, (median $=80)$. Nine patients were contrast-enhanced in preoperative MRI, and intraoperatively stained by gross labeling with fluorescein sodium. The control group consisted of 12 patients, including five female and seven male patients, whose ages ranged between 41 and 64 years. The mean age was $51.8 \pm 6.8$ (median 50.5 years). Duration of disease ranged between 20 to 180 days $($ mean $=62$ days). Preoperative KPS was $79.2 \pm 10.8$ (median 80). KPS scores were re-evaluated one week postoperatively. In all patients, the extent of resection was identified by MRI less than one week after surgery. Gross-total resection is defined as resection without visual residual enhancing tumor [5]. To evaluate the effect of GTR on progression free survival, patients were divided into two groups according to the extent of resection postoperatively.

\section{Surgical Procedure and Policy}

Craniotomy was performed after general anesthesia. After the dura at the craniotomy site was opened, fluorescein sodium (diluted into $1 \%, 5 \mathrm{ml}$; Alcon, USA, 5ml: 500mg, import drug ID was H20090507) was injected intravenously. Vital signs were monitored for 15 minutes. Following confirmation that no skin rash was present, $10 \mathrm{ml}$ of high dose fluorescein sodium (diluted into $10 \%, 15-20 \mathrm{mg} / \mathrm{kg}$ ) was injected intravenously without abnormalities. The staining of tumor began to develop from light yellow to deep yellow after 5 to 10 minutes, which was visible to the naked eye. The visible yellow stained region of the tumor was resected entirely using a standard microsurgical procedure with white-light illumination. Following surgery, resected tissue with three-color degrees was submitted to histology for further examination.

\section{Statistical Analysis}

The Fisher's Exact Test was used to evaluate the significance in the difference between GTR rates in patients of the study group who underwent fluorescein sodium and those who did not. Student's T-Test was used to evaluate the significance of age and KPS score pre- and post-operation in difference between the study group and control group as well as the difference towards progressive free survival time between GTR and non-GTR group. The Fisher's Exact Test was performed with SPSS 13.0 software. And Student's T-Test was performed with EXCEL software. Significance was assigned at a probability value of less than 0.05 .

\section{Results}

\section{Fluorescein Sodium Staining}

Three color degrees of staining were observed that were deep yellow, light yellow and negatively stained. Normal brain parenchyma remained unstained. Tumor stained with yellow was easily identified from the normal brain parenchyma (Fig. 1A-C). Most of the patients $(n=9)$ within the study group $(n=10)$ had tumors that were positively stained with fluorescein sodium. The case that did not have a pos- 
itively stained tumor was subsequently identified to have a grade II glioma. Meanwhile, we reviewed pre-operative enhanced MRI and found that the edges of the tumor were not clearly enhanced.

\section{Extent of Surgery}

Fluorescence-guidance of fluorescein sodium can increase the GTR rate. Ten patients received fluorescein sodium. Of the ten patients, GTR was completed in eight patients (GTR rate $80 \%$ ) and STR was completed in two patients $(20 \%)$. In the control group, there were only four patients who had received GTR (GTR rate $33.3 \%$ ) and eight patients who had received STR $(66.7 \%)$. There was a significant difference in GTR rate between two groups (Fisher's Exact Test $p=0.047$ ).

\section{Post-operation}

Postoperatively, patients accepted general treatment such as reducing intracranial pressure, preventing infection and controlling epilepsy. Yellow staining of sclera, skin and urine disappeared in approximately 24 hours. There were no abnormal changes observed during routine blood or urine examination or with the liver and kidney function test. The extent of tumor resection was identified by enhanced MRI one week postoperatively. All the patients were treated with $\gamma$-knife radiotherapy with or without postoperative temozolomide chemotherapy.

\section{Histological examination}

In the study group, four astrocytomas (WHO grade II), three anaplastic astrocytomas (WHO grade III) and three GBM (WHO grade IV) were identified. The characteristics of tumor tissue from the three yellow stained regions were different. In the deeply stained yellow region (Fig. 2A, Fig. 2D), tumor cells were densely packed, significantly atypical, multinuclear, and mitotic with necrosis visible in the tumor cells and necrotic degeneration of the surrounding tumor tissue accompanied with vascular endothelial cell proliferation. The sample from faintly yellow stained area (Fig. 2B, Fig. 2E), had a low density of scattered tumor cells and scattered vascular endothelial proliferation. From the negatively stained area (Fig. 2C, Fig. 2F), there were rare or no tumor cells, no endothelial cell proliferation or visible necrosis. In the control group, there were five astrocytomas (WHO grade II), three astrocytomas (WHO grade III) and four GBM (WHO grade IV).

\section{Postsurgical complications}

Three cases in the study group and four patients in the control group experienced transient verbal and motor dysfunction, and gradually recovered after symptomatic treatment. One patient in the control group had permanent hemiplegia. Follow-up in twenty patients ranged from six to ten months after the operation. In the study group, eight patients confirmed they were free of symptoms with no evidence of recurrence. Two patients from the study group recurred, but are still alive. In the control group, five patients had recurrence including two patients that died after their glioma recurred. The mean progressive free survival time was more than 7.2 months in the study group and more than 5.4 months in control group. In addition, it was more than 7.4 months in GTR group and 4.8 months in non-GTR group. There was a significant difference in the progressive free survival time between the study group and control group (Student t-test $p=0.033$ ) as well as between GTR group and non-GTR group (Student t-test $p=0.0001$ ). Post-operative KPS score was $75.5 \pm 8.3$ (median 77.5) and $75.8 \pm 10.2$ (median 80 ) in the study group and control group, respectively. There was no significant decrease between the postoperative KPS score and preoperative KPS score (Student t-test $p>0.05$ ). The results are summarized in Tables 1 and 2.
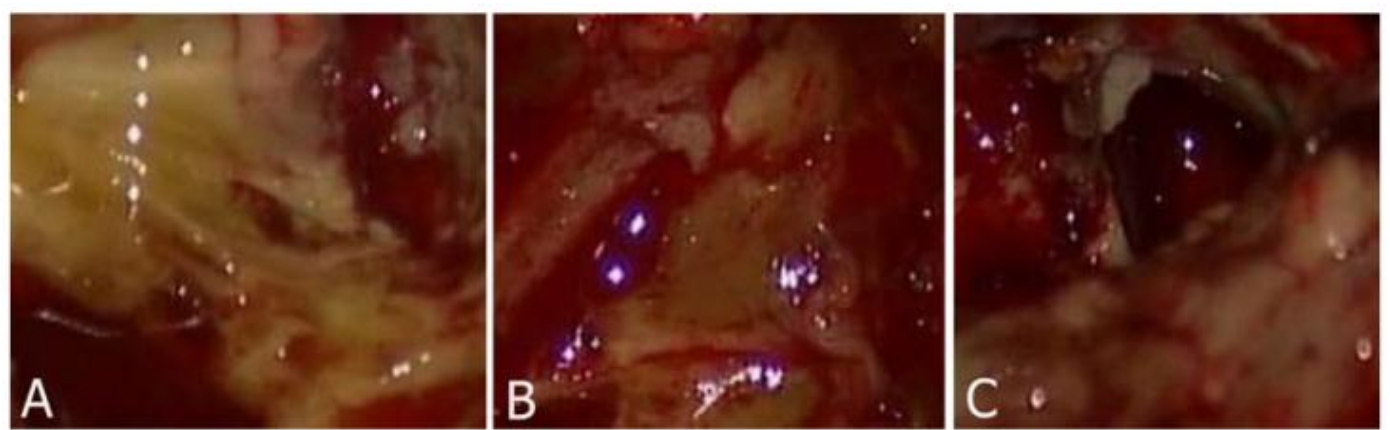

Fig. I: A-B: Tumor tissue was stained by fluorescein sodium presenting deep to light yellow color. C: Normal parenchyma was not stained. 


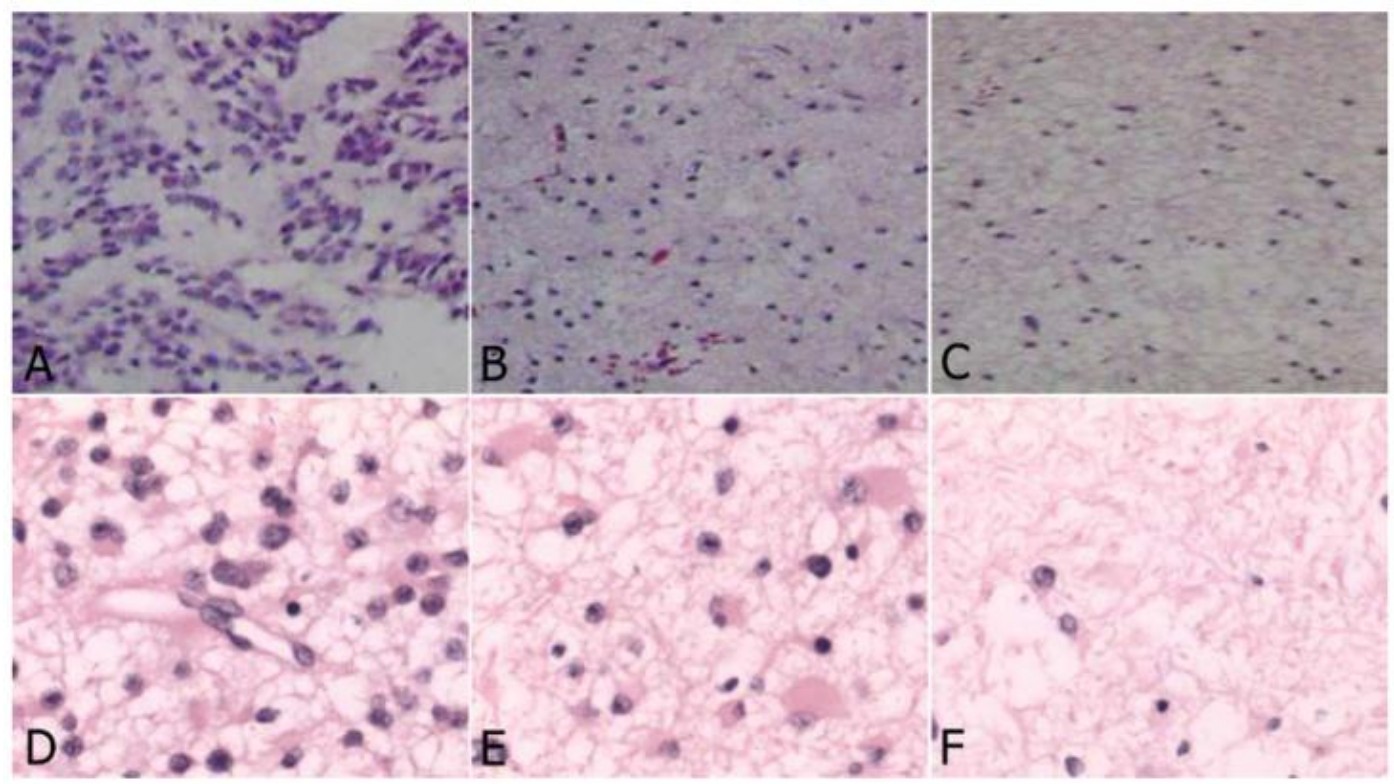

Fig. 2: Histology of glioma tissue in study group $(\mathrm{HE}$, original magnification×200). A-C: High grade glioma. D-F: Low grade glioma. A and D: Deep yellow stained region: dense tumor cells, which were significantly atypical, multinuclear, mitosis, with prominent endothelial proliferation; B and E: Light stained region: less dense tumor cells with scattered endothelial proliferation; C and F: Negative stained region: very rare tumor cells without endothelial proliferation.

Table I. Summary of the characteristics of two groups

\begin{tabular}{|c|c|c|}
\hline Group & Study & Control \\
\hline number of patients & 10 & 12 \\
\hline \multicolumn{3}{|l|}{ sex } \\
\hline $\mathrm{fm}$ & 4 & 5 \\
\hline $\mathrm{m}$ & 6 & 7 \\
\hline \multicolumn{3}{|l|}{ age(yrs) \# } \\
\hline range & 31 to 61 & 41 to 64 \\
\hline mean & $50.7 \pm 10.4$ & $51.8 \pm 6.8$ \\
\hline median & 50 & 50.5 \\
\hline \multicolumn{3}{|l|}{ Location } \\
\hline non-eloquent area & 7 & 9 \\
\hline eloquent area & 3 & 3 \\
\hline \multicolumn{3}{|l|}{ symptom/sign } \\
\hline intermittent headache & 7 & 8 \\
\hline hemiparesis and/or aphasia & 4 & 6 \\
\hline seizure & 2 & 3 \\
\hline \multicolumn{3}{|l|}{ KPS score\#\# } \\
\hline preoperative & $78.0(9.2)$ & $79.2(10.8)$ \\
\hline postoperative & $75.5(8.3)$ & $75.8(10.2)$ \\
\hline \multicolumn{3}{|l|}{ extent of tumor resection } \\
\hline $\mathrm{GTR}^{*}$ & 8 & 4 \\
\hline STR & 2 & 8 \\
\hline \multicolumn{3}{|l|}{ Follow up 6-10 months } \\
\hline Recurrence & 2 & 7 \\
\hline Progressive free survival (months) ** & 7.2 & 5.4 \\
\hline
\end{tabular}

\# No significant difference between two groups, Student t-test $(\mathrm{p}>0.05)$

\#\# No significant difference between preoperative and postoperative KPS score in the two groups, Student $t$-test ( $p>0.05)$.

* Significant difference between two groups, Fisher's Exact Test $(p=0.047)$

** Significant difference between two groups, Student t-test $(\mathrm{p}=0.033)$ 
Table 2. Progressive free survival between GTR and non-GTR group

\begin{tabular}{lll}
\hline Groups & GTR & Non-GTR \\
\hline Number of patients & 12 & 10 \\
* Progressive free survival (months) & 7.4 & 4.8 \\
\hline
\end{tabular}

* Significant difference between two groups, Student $\mathrm{t}$-test $(\mathrm{p}=0.0001)$

\section{Discussion}

The boundary between gliomas and brain parenchyma is difficult to discern due to their characteristic invasiveness, making it more difficult to resect these tumors completely. There is a growing amount of evidence indicating that the extent of tumor resection is a major factor dictating the postoperative survival of patients $[2-4,7,10]$. Over the past six decades, a variety of innovative techniques have been developed by neurosurgeons to increase the efficacy of resecting primary malignant brain tumors such as intraoperative ultrasound, neuronavigation, intraoperative MRI, cortical stimulations and fluorescence-guided resection [11, 12]. Among these methods, fluorescence-guided resection has shown great promise because it allows intraoperative visualization of tumor boundaries, which are otherwise difficult to recognize $[1,5,6,13-17]$.

Though the significant increase in the extent of resection using high dose of fluorescein sodium, the efficiency of using fluorescein sodium is still controversial, as some studies have described little color contrast between the tumor and surrounding brain tissue $[8,9]$. We observed that lightly stained areas in eight patients from the study group included part of adjacent edematous areas, which were outside of enhanced tumor areas in preoperative MRI. Similarly, the vague staining in the boundary areas of some cases was also seen in studies using 5 -aminolevulinic acid (5-ALA), another fluorescent agent used in glioma resection. Using 5-aminolevulinic, Ricardo et al [18] found that these vague areas corresponded with areas where tumor cells infiltrate brain tissue potentially beyond the gadolinium-enhancing border of the tumor, which was not visible in postoperative MRI [19]. We hypothesized that this mechanism may also apply when using fluorescein sodium. Obviously, resection of these areas may be beneficial for reducing recurrence and malignant progression. Many other studies have indicated that utilizing high doses of fluorescein sodium was a useful intraoperative fluorescence-guide agent even without any specific visualization equipment $[5,7,20]$.

Shinoda et al [7] reported that GTR was signifi- cantly increased by using fluorescein sodium to visualize brain tumors. In this study, high doses of fluorescein sodium $(20 \mathrm{mg} / \mathrm{kg})$ were applied intravenously into thirty-two patients resulting in an achieved GTR in twenty-seven patients (84.4\%), a significantly greater difference in GTR compared with controls. Koc et al [5], performed significantly higher rates of GTR by using fluorescein-sodium-guidance. In their study, forty-seven patients were recruited into the study group, and GTR was achieved in thirty-nine patients $(83 \%)$ compared with eighteen patients $(54.5 \%)$ in the control group. They also investigated the factors affecting survival, including GTR, KPS and age, which were significantly associated with prognosis. In both of these studies, the utility of high-dose fluorescein sodium in surgical resection of low-grade glioma was not evaluated. Combined approaches based on fluorescence agents and other visualization techniques seem to be more effective [14, 21].

In the present study, because we used a high dose of fluorescein sodium $(15-20 \mathrm{mg} / \mathrm{Kg})$ there was no need for the use of a special surgical microscopic. The fluorescein sodium stained portions of the tumor were seen directly by the naked eye. The utility of fluorescein sodium in the operation of glioma was reevaluated and led to an improved GTR rate. Although the number of patients in our study was relatively small the GTR rate of the study group corresponded to previous data published in the literature [7]. Notably, the three GTR cases of low-grade glioma were clearly stained, which corresponded with preoperative enhanced MRI. Although one low-grade glioma without clear staining was not enhanced in preoperative MRI, we were able to resect the vaguely stained areas in non-eloquent region of the three low-grade glioma cases present in the study group. Resection of the low-grade gliomas in the study group resulted in a progressive free survival time of more than six months, indicating that fluorescein sodium may be useful in the treatment of low-grade glioma. Also, resection of the vaguely stained areas could be associated with reduced recurrence and malignant progression. In the control group, we identified five STR cases that were confirmed by using postoperative MRI. Originally, these tumors were mistakenly iden- 
tified as GTR intraoperatively. Because the tumors in the study group were completely resected, we hypothesized that fluorescein sodium may help neurosurgeons to complete GTR of glioma, which would instead be STR without fluorescein sodium guidance. Since this study was not designed to evaluate survival, we were unable to accurately calculate a median survival time, especially since there are patients remaining alive in each group. However, there was a significant trend towards progressive free survival time between the study group and control group (Student's T-Test $p=0.033$ ) as well as between the GTR group and non-GTR group (Student's T-Test $p=0.0001$ ) based on results obtained during postoperative follow up. Homogenization of other factors that may influence survival resulted in us being able to conclude that the benefit was due to the increased GTR rate. Histological examination of the tumor in the study group indicated that the yellow stained location was consistent with most of the glioma tissue. Exclusive staining of glioma tumor tissue with fluorescein sodium instead of normal brain tissue may be associated with the blood brain barrier disruption induced by the invasiveness of glioma [7], facilitating easy fluorescein sodium penetration into the tumor lesion. In both high-grade and low-grade stained areas, the degree of fluorescein sodium staining was positively correlated with tumor cell density. In positively stained areas, there were greater numbers of tumor cells (Fig. 2A-B, Fig. 2D-E) compared to the non-stained areas of the brain where almost none tumor cells were observed (Fig. 2C, Fig. 2F). These staining patterns indicated that resecting all of the positively yellow stained areas could result in achieving GTR. Of the ten patients in the study group, only one did not have significant fluorescein sodium staining of the tumor due to a disrupted BBB detected in the preoperative MRI.

Fluorescein sodium is a fluorescence agent commonly used in clinical examinations such as ophthalmoscopic examinations and visualization of the retina [7]. Use of fluorescein sodium is very safe although an anaphylactic reaction was previously reported in one case report [22]. In our study, before injection of high dose fluorescein sodium, we performed allergy testing and monitored the vital signs in the event of an adverse reaction. Because radiotherapy and chemotherapy was confirmed to be significant prognostic factors for survival $[23,24]$, in our study, all patients were advised to receive $\gamma-\mathrm{knife}$ radiotherapy with or without chemotherapy of temozolomide postoperatively.

\section{Conclusion}

High dose of fluorescein sodium was indeed a useful agent for the resection of glioma. It is a simple, convenient, effective and inexpensive technique compared with other techniques. Although GTR was achieved in most glioma patients, some residual tumor cells remained, necessitating the need for postoperative adjuvant therapy. Because fluorescein sodium stained significantly in cases of BBB disruption, we suggest that the fluorescein sodium is best used in the cases with BBB disruption.

\section{Acknowledgments}

We would like to thank Melanie R Rutkowski for critical reading and editing of the manuscript.

\section{Competing Interests}

The authors have declared that no competing interest exists.

\section{References}

1. Babu R,Adamson C. Fluorescence-Guided Malignant Glioma Resections. Curr Drug Discov Technol. 2012; [Epub ahead of print].

2. Shinoda J, Sakai N, Murase S, et al. Selection of eligible patients with supratentorial glioblastoma multiforme for gross total resection. J Neurooncol. 2001; 52: 161-71.

3. Stummer W, Reulen HJ, Meinel T, et al. Extent of resection and survival in glioblastoma multiforme: identification of and adjustment for bias. Neurosurgery. 2008; 62: 564-76.

4. Tykocki T, Michalik R, Bonicki W, et al. Fluorescence-guided resection of primary and recurrent malignant gliomas with 5-aminolevulinic acid. Preliminary results. Neurol Neurochir Pol. 2012; 46: 47-51.

5. Koc K, Anik I, Cabuk B, et al. Fluorescein sodium-guided surgery in glioblastoma multiforme: a prospective evaluation. Br J Neurosurg. 2008; 22: 99-103.

6. Kabuto M, Kubota T, Kobayashi H, et al. Experimental and clinical study of detection of glioma at surgery using fluorescent imaging by a surgical microscope after fluorescein administration. Neurol Res. 1997; 19: 9-16.

7. Shinoda J, Yano H, Yoshimura S, et al. Fluorescence-guided resection of glioblastoma multiforme by using high-dose fluorescein sodium. Technical note. J Neurosurg. 2003; 99: 597-603.

8. Ichioka T, Miyatake S, Asai N, et al. Enhanced detection of malignant glioma xenograft by fluorescein-human serum albumin conjugate. J Neurooncol. 2004; 67: 47-52.

9. Kuroiwa T, Kajimoto Y, Ohta T. Development of a fluorescein operative microscope for use during malignant glioma surgery: a technical note and preliminary report. Surg Neurol. 1998; 50: 41-9.

10. Buckner JC. Factors influencing survival in high-grade gliomas. Semin Oncol. 2003; 30: 10-4.

11. Litofsky NS, Bauer AM, Kasper RS, et al. Image-guided resection of high-grade glioma: patient selection factors and outcome. Neurosurg Focus. 2006; 20: E16.

12. Hammoud MA, Ligon BL, elSouki R, et al. Use of intraoperative ultrasound for localizing tumors and determining the extent of resection: a comparative study with magnetic resonance imaging. J Neurosurg. 1996; 84: 737-41.

13. Ritz R, Daniels R, Noell S, et al. Hypericin for visualization of high grade gliomas: first clinical experience. Eur J Surg Oncol. 2012; 38: 352-60.

14. Feigl GC, Ritz R, Moraes M, et al. Resection of malignant brain tumors in eloquent cortical areas: a new multimodal approach combining 5-aminolevulinic acid and intraoperative monitoring. J Neurosurg. 2010; 113: 352-7.

15. Eljamel MS, Goodman C,Moseley H. ALA and Photofrin fluorescence-guided resection and repetitive PDT in glioblastoma multiforme: a single centre Phase III randomised controlled trial. Lasers Med Sci. 2008; 23: 361-7. 
16. Willems PW, Taphoorn MJ, Burger $\mathrm{H}$, et al. Effectiveness of neuronavigation in resecting solitary intracerebral contrast-enhancing tumors: a randomized controlled trial. J Neurosurg. 2006; 104: 360-8.

17. Moore GE, Peyton WT,et al. The clinical use of fluorescein in neurosurgery; the localization of brain tumors. J Neurosurg. 1948; 5: 392-8.

18. Diez Valle R, Tejada Solis S, Idoate Gastearena MA, et al. Surgery guided by 5-aminolevulinic fluorescence in glioblastoma: volumetric analysis of extent of resection in single-center experience. J Neurooncol. 2011; 102: 105-13.

19. Stummer W, Novotny A, Stepp H, et al. Fluorescence-guided resection of glioblastoma multiforme by using 5-aminolevulinic acid-induced porphyrins: a prospective study in 52 consecutive patients. J Neurosurg. 2000; 93: 1003-13.

20. Okuda T, Kataoka K,Taneda M. Metastatic brain tumor surgery using fluorescein sodium: technical note. Minim Invasive Neurosurg. 2007; 50: 382-4.

21. Panciani PP, Fontanella M, Garbossa D, et al. 5-aminolevulinic acid and neuronavigation in high-grade glioma surgery: results of a combined approach. Neurocirugia (Astur). 2012; 23: 23-8.

22. Dilek $O$, Ihsan A,Tulay $H$. Anaphylactic reaction after fluorescein sodium administration during intracranial surgery. J Clin Neurosci. 2011; 18: 430-1.

23. Mineo JF, Bordron A, Baroncini M, et al. Prognosis factors of survival time in patients with glioblastoma multiforme: a multivariate analysis of 340 patients. Acta Neurochir (Wien). 2007; 149:245-52.

24. Freyschlag CF, Smolczyk DR, Janzen E, et al. Prolonged administration of temozolomide in adult patients with anaplastic glioma. Anticancer Res. 2011; 31: 3873-7. 